\title{
Comparison of centralized, distributed and hierarchical model predictive control schemes for electromechanical oscillations damping in large-scale power systems
}

\author{
Da Wang, Mevludin Glavic, Louis Wehenkel* \\ Department of EEGCS, University of Liège, Belgium
}

\section{A R T I C L E I N F O}

\section{Article history:}

Received 22 April 2013

Received in revised form 19 November 2013 Accepted 2 January 2014

\section{Keywords:}

Model predictive control

Electromechanical oscillations damping

Wide-area measurement systems

\begin{abstract}
A B S T R A C T
The paper investigates the feasibility of applying Model Predictive Control (MPC) as a viable strategy to damp wide-area electromechanical oscillations in large-scale power systems. First a fully centralized MPC scheme is considered, and its performances are evaluated first in ideal conditions and then by considering state estimation errors and communication delays. This scheme is further extended into a distributed scheme with the aim of making it more viable for very large-scale or multi-area systems. Finally, a robust hierarchical multi-area MPC scheme is proposed, introducing a second layer of MPC based controllers at the level of individual power plants and transmission lines. Simulations are carried out using a 70-bus test system. The results reveal all three MPC schemes as viable solutions to supplement existing controllers in order to improve the system performance in terms of damping. The hierarchical scheme is the one combining the best performances in nominal conditions and the best robustness with respect to partial component failures and various modeling and measurement errors.
\end{abstract}

(c) 2014 Elsevier Ltd. All rights reserved.

\section{Introduction}

Some characteristics of modern large-scale electric power systems, such as long transmission distances over weak grids, highly variable generation patterns and heavy loading, tend to increase the probability of appearance of sustained wide-area electromechanical oscillations. The term "wide-area" is used here to emphasize the possible co-existence of local and inter-area oscillation modes of different frequencies that might appear simultaneously in different parts of large-scale systems. Such oscillations threaten the secure operation of power systems and if not controlled efficiently can lead to generator outages, line tripping and large-scale blackouts [1-3]. Current automatic control systems, designed to address low-frequency oscillations, are mostly based on very local control strategies realized through Power System Stabilizers (PSSs) and FACTS devices.

The emergence of new technological solutions such as synchronized phasor measurement devices and improved communication infrastructures enable the development of Wide Area Measurement Systems (WAMS) [4] and the design of new types of controllers [5]. Such controllers may be designed from the perspective of the whole system, focus on a wider spectrum of oscillation modes and offer improvements with respect to current local control strategies.

\footnotetext{
* Corresponding author.

E-mail address: l.wehenkel@ulg.ac.be (L. Wehenkel).
}

In this paper, such controllers to damp power system electromechanical oscillations are proposed, based on the following observations:

- The control rules and parameters of current automatic control systems are usually fixed and determined in off-line studies using time-domain simulations, Prony or eigenanalysis [1,3], and are based on local voltages, generator speeds or line powers as inputs. Increasing uncertainties brought by renewable generation, and the growing complexity resulting from new power flow control devices, make the robustness of these designs become questionable, yielding the need for more efficient, adaptive, and more widely coordinated control schemes.

- A promising option would be a control strategy able to automatically adjust its control actions to the changing nature of the system. Since power system dynamics can be quite accurately modeled [4], and given the recent progresses in largescale optimization, a natural idea is to apply MPC [6] to design such control strategies.

- MPC is a proven technique with numerous real-life applications in different engineering fields [7] and it may be designed in different ways: centralized [6], distributed [8,9] and hierarchical [9].

- Among existing MPC formulations [6,7], those proven in other fields [7], i.e. linear MPC formulation, should be considered first. 
In this work, a series of MPC controllers is considered, which can be activated by faults or special local states, or run all the time. These MPC controllers collect system states at a given time interval, compute supplementary control inputs for existing damping controllers, like PSS and Thyristor Controlled Series Compensator (TCSC), and superimpose these supplementary inputs on these devices' own inputs in order to optimize and coordinate their control effects. Three different MPC schemes are considered (centralized, distributed, and hierarchical), and their performances are compared on a medium sized power system.

The rest of the paper is organized as follows. After a synthetic review of previous works in Section 2, Section 3 describes the linear MPC formulation that we use, and Section 4 proposes three different control architectures. The used test system and simulation parameters are given in Section 5, while results are discussed in Section 6 . Section 7 offers some conclusions.

\section{Related works}

MPC considerations in power systems include security constrained optimal power flow [10], coordinated secondary voltage control [11], thermal overload alleviation [12,13], voltage control [14-16], transient stability [17], oscillations [18-24], and automatic generation control $[25,26]$. The works dealing with MPC applications to control electromechanical oscillations of power systems can be broadly classified into the three categories discussed below.

MPC to control a single device. One of the earliest applications is presented in [20] where generalized predictive control [6] is used to switch capacitors for damping power system oscillations. The control is computed by minimizing a quadratic cost function combining local system outputs and rates-of-change of control over the prediction horizon. An MPC for step-wise series reactance modulation of a TCSC to stabilize electromechanical oscillations is presented in [21], where a reduced two-machine model of the power system is used and updated using local measurements. Defining deviations of the predicted outputs from references and control input increments as an objective function, Ref. [22] proposed a model predictive adaptive controller based on an equivalent model to damp inter-area oscillations in a four-generator system.

Centralized system-wide MPC. Refs. [18,19] present a widearea MPC to control low-frequency oscillations. A bank of linearized system models is used with the assumption that the actual system response can be represented by a suitable combination of a finite number of linearized models. For each model in the bank an observer-based state feedback controller is designed a priori and MPC is formulated to optimize the weights for individual controllers in the bank. An MPC scheme introduced in [23] coordinates local control devices (PSS, TCSC, and static var compensators (SVC)) to damp wide-area electromechanical oscillations. The MPC scheme is based on a linearized discrete-time state space model of the power system combined with a quadratic objective function.

Distributed multi-area MPC. Distributed MPC for electromechanical oscillations damping is considered in [24]. The problem is formulated using a context-driven decomposition of control areas. An MPC controller is assigned to each control area and three coordination schemes are considered: an implicit scheme where the overall system stability emerges from individual MPC controllers, and two explicitly coordinated ones.

The approaches considered in this paper put their emphasis on the need to coordinate existing damping controllers in the least intrusive way and propose the MPC approach as a paradigm for coordination. This work extends previous ones of the same authors $[23,24]$ by introducing a hierarchical MPC control scheme and showing its superior performances through comparison with two other MPC based schemes and by considering the centralized MPC as the benchmark.

\section{Generic MPC framework formulation}

The principle of MPC can be shortly summarized as follows. At any time, the MPC algorithm uses the collected measurements, a model of the system and a specification of the control objective to compute an optimal open-loop control sequence over a specified time horizon. The first-stage controls are applied to the system. At the next time step, as soon as measurements (or model) updates are available, the entire procedure is repeated by solving a new optimization problem with the time horizon shifted one step forward [6]. In this section, MPC formulation is first provided in terms of the model (prediction equations) and optimal control problem (objective function and constraints). Then a way to take into account data acquisition errors and time delays due to computation and communication resources, is proposed.

\subsection{Discrete time linearized dynamic system model}

MPC algorithms, considered in this work, are based on a statespace model of a multi-machine power system in the form of the following linearized continuous time model:

$$
\left\{\begin{array}{l}
\dot{x}=A_{c} x+B_{c} u \\
y=C_{c} x
\end{array}\right.
$$

where $x \in R^{m_{x}}$ is a vector of state variables modeling also the already existing controllers, $u \in R^{m_{u}}$ is a vector of supplementary MPC inputs, and $y \in R^{m_{y}}$ is a vector of performance measurements used by MPC (outputs).

Next, from Eq. (1) the transition at time $t$ for a small step of $\delta$ seconds is inferred by [6]:

$$
\left\{\begin{array}{l}
x(t+\delta)=\left(\delta A_{c}+I\right) x(t)+\delta B u(t) \\
y(t)=C x(t)
\end{array}\right.
$$

yielding a discrete-time dynamic (for time steps $t+i \delta$ ) given by:

$x[i+1]=A x[i]+B u[i]$

$y[i]=C x[i]$.

with : $A=\delta A_{c}+I ; B=\delta B_{c} ; C=C_{c}$.

\subsection{MPC formulation as a quadratic programming problem}

At time $t=k \Delta t$ (system states are collected every $\Delta t$ seconds), based on the estimation $\hat{x}(k \Delta t)=\hat{x}[k \mid k]$ of the current system states and on the system model, the predicted output $\hat{y}(k \Delta t+i \delta)=\hat{y}[k+i \mid k]$ over the next horizon $N_{i} \delta$ is obtained by iterating Eq. (3) $i$ times, $i=0,1,2, \ldots N_{i}-1$.

$$
\left[\begin{array}{c}
\hat{y}[k+1 \mid k] \\
\hat{y}[k+2 \mid k] \\
\vdots \\
\hat{y}\left[k+N_{i} \mid k\right]
\end{array}\right]=P_{x} \hat{x}[k \mid k]+P_{u}\left[\begin{array}{c}
u[k \mid k] \\
u[k+1 \mid k] \\
\vdots \\
u\left[k+N_{i}-1 \mid k\right]
\end{array}\right]
$$

where $P_{x}$ and $P_{u}$ are given by

$P_{x}=\left[\begin{array}{c}C A \\ C A^{2} \\ \vdots \\ C A^{N_{i}}\end{array}\right], P_{u}=\left[\begin{array}{cccc}C B & 0 & \ldots & 0 \\ C A B & C B & \ldots & 0 \\ \vdots & \vdots & \ddots & \vdots \\ C A^{N_{i}} B & C A^{N_{i}-2} B & \ldots & C B\end{array}\right]$

Using these equations, the following quadratic optimization problem is solved at every time step [6]: 
$\min _{u[\cdot]}\left\{\sum_{i=0}^{N_{i}-1}\left(\hat{y}[k+i+1 \mid k]-y_{r}\right)^{T} Q\left(\hat{y}[k+i+1 \mid k]-y_{r}\right)\right\}$

subject to linear inequality constraints:

$u_{\min } \leqslant u[k+i \mid k] \leqslant u_{\max }, \quad i=0,1,2, \ldots, N_{i}-1$.

$z_{\min } \leqslant z[k+i+1 \mid k] \leqslant z_{\max }, \quad i=0,1,2, \ldots, N_{i}-1$.

where $Q$ is a symmetric positive definite matrix of weights, $y_{r}$ is the vector of performance targets, and $z$ is a vector of constrained operation variables. During one step of length $\Delta t$, it is assumed that $u[k+i \mid k]$ remains constant.

\subsection{Consideration of state estimation (SE) errors and time delays}

MPC uses the estimated system state $\hat{x}[k \mid k]$ from a SE as initial value for computing its optimal decisions. Consequently, the SE imprecision may have a detrimental effect on the MPC controller's decisions. In order to compensate for this imprecision, at time $k \Delta t$, the difference $d[k \mid k]$ between the actual output and its predicted value is calculated to correct Eq. (4) as:

$$
\left[\begin{array}{c}
\hat{y}[k+1 \mid k] \\
\hat{y}[k+2 \mid k] \\
\vdots \\
\hat{y}\left[k+N_{i} \mid k\right]
\end{array}\right]=P_{x} \hat{x}[k \mid k]+P_{u}\left[\begin{array}{c}
u[k \mid k] \\
u[k+1 \mid k] \\
\vdots \\
u\left[k+N_{i}-1 \mid k\right]
\end{array}\right]+\left[\begin{array}{c}
I \\
I \\
\vdots \\
I
\end{array}\right] d[k \mid k]
$$

where

$d[k \mid k]=y[k \mid k]-C \hat{x}[k \mid k-1]$

It is assumed that $d[k \mid k]$ is refreshed each time a set of new SE results is collected, and then remains unchanged over the entire prediction horizon used to compute the controls.

Delays in measurements' acquisition, computation and applying MPC decisions, are involved in the MPC implementation [6]. It is therefore important to assess the impact of such delays on the performances of the proposed MPC schemes. The impact is studied by assuming that all measurements are taken synchronously, and then exploited with a common delay [23,24].

\section{Proposed MPC schemes}

\subsection{Centralized scheme (adapted from [23])}

Centralized MPC scheme is described in more details in [23]. In this scheme, the MPC controller obtains a complete system model from the energy management systems, which can be refreshed from time to time following changes of the load level, the generation schedule and the grid topology. It collects system states from a SE and WAMS [4] at discrete measurement times $k \Delta t$. It then computes an open loop sequence of the control variable $u$ over a certain time horizon composed of $H_{p}$ steps of length $\Delta t\left(H_{p} \Delta t=N_{i} \delta\right)$. It applies the controls determined for the first period of $\Delta t$ seconds to local controllers (LCs). The cost-function (5) is formulated as the sum of square deviations of all generator speeds from the nominal frequency.

\subsection{Distributed scheme (adapted from [24])}

Wide-area power system oscillations tend to appear in very large-scale systems involving many different subsystems managed by different transmission system operators (TSOs) [2]. It is often practically not feasible to handle these problems with a fully centralized approach. On the other hand, reliability/vulnerability considerations may suggest that even in a system where a fully centralized control scheme would be feasible, it is not necessarily desirable to do so. Consequently, it is of interest to study distributed MPC schemes that could be more viable and easier to implement. As with any other distributed control scheme, two problems are to be solved, namely decomposition and coordination.

\subsubsection{Control problem decomposition}

A large-scale control problem can be decomposed into subproblems by the following two main approaches:

- Problem-driven: construction of a global system model followed by an optimal decomposition into subsystems according to structural properties of the system and the control problem under consideration [29].

- Context-driven: the decomposition of the whole system is imposed by contingent constraints, and hence the construction of the local control schemes has to follow the already given decomposition.

Considering information-exchange restrictions in certain power grids, and organizational barriers, it is quite difficult to construct an exact system-wide model, and even if this is possible, it would be difficult to impose significant changes within the existing control structures. Therefore it is prefered to consider in this paper a context-driven decomposition of control areas [26].

Let us consider a two-area system, shown in Fig. 1. Notice that this system is decomposed a priori into two control areas, linked by tie-lines allowing to exchange power. A control system only able to act on one of the two subsystems could view the rest of the system by an equivalent dynamic load in order to compute its control inputs. Accordingly, the control objective of Eq. (5) is rewritten as the simultaneous and parallel resolution of the following area-wise problems (subscript $n$ refers to area $n$ ):

$\min _{u_{n}[\cdot]}\left\{\sum_{i=0}^{N_{i}-1}\left(\hat{y}_{n}[k+i+1 \mid k]-y_{r . n}\right)^{T} Q_{n}\left(\hat{y}_{n}[k+i+1 \mid k]-y_{r . n}\right)\right\}$

subject to linear inequality constraints:

$$
\begin{aligned}
& u_{\text {min. } n} \leqslant u_{n}[k+i \mid k] \leqslant u_{\text {max.n }}, \quad i=0,1,2, \ldots, N_{i}-1 . \\
& z_{\text {min. } n} \leqslant z_{n}[k+i+1 \mid k] \leqslant z_{\text {max. } n}, \quad i=0,1,2, \ldots, N_{i}-1 .
\end{aligned}
$$

In this scheme, each local MPC controller solves its optimization problem using a detailed model of its own area and a possibly very rough model of the remaining areas (typically a black box model). It then sends the first inputs of the computed optimal control sequence to the controllers under its responsibility, and observes the resulting effects to proceed.

\subsubsection{Coordination of controls of subsystems}

Since distributed MPC controllers act in the same system, coordination is needed in order to achieve satisfactory performances $[8,25,12]$. They could negotiate/exchange useful information in or-

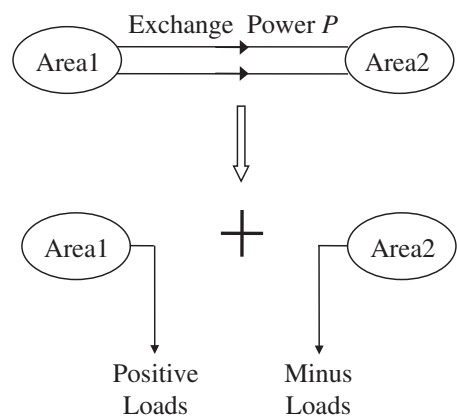

Fig. 1. Decomposition of an interconnected grid into two subsystems. 
der to improve global performance. However, in very large-scale systems, there may be many remote areas, thousands of kilometers away from each other and separated by many intermediate areas. Communicating and negotiating between all these areas would require supporting communications infrastructure. In today's large interconnections the lack of communications infrastructure is the main obstacle for implementing advanced control schemes. Upgrade of this infrastructure is costly and will remain an issue at least in near future. Consequently, instead of explicit communication/negotiation, an implicit coordination scheme is used in this context. Specifically, each subsystem tries to solve its own oscillation problem and the overall system stability emerges from these area-wise controls [25]. As far as the considered scheme is concerned, each area-wise MPC controller adjusts inputs of damping controllers, under its authority, so as to minimize deviations of individual generators' angular speeds from their nominal values, while ensuring that all constraints are satisfied.

\subsection{Hierarchical and distributed two-layer scheme}

A hierarchical structure is often used to coordinate local controllers to improve global control effects. In [5], a wide-area central controller is responsible to decouple subsystems dynamics and calculate the interactions among them for lower-level local controllers. Ref. [27] proposes a two-loop hierarchical structure: a local loop based on a machine speed signal and a global loop based on a differential frequency between two remote areas. The total PSS signal applied to the machine voltage reference is the sum of the control components of these two loops.

A hierarchical MPC scheme based on the distributed one is proposed aiming at improved control robustness, e.g. in the case of failure of one of the area-wise controllers. In addition to MPC controllers operating at the level of whole areas (subsystems), a lower level layer of independent MPC controllers attached to $N_{m}$ basic control devices is added (Fig. 2).

\subsubsection{MPC in the lower level}

Compared with MPC controllers in the upper level, MPC controllers in the lower level only concern dynamic behaviors of one generator or tie-line. These controllers need less time to measure, compute and apply their decisions, so that they can update their control decisions more frequently following changes of system states, and thus approach their control targets in a possibly better way. In addition, when upper MPC controllers cannot work normally, lower MPC controllers are designed to work independently with the aid of internal control objectives. There are an exciter, a PSS and a turbine governor on each generator. A generator model used is the one including the effects of sub-transient circuits. These devices are modeled by the following equations:

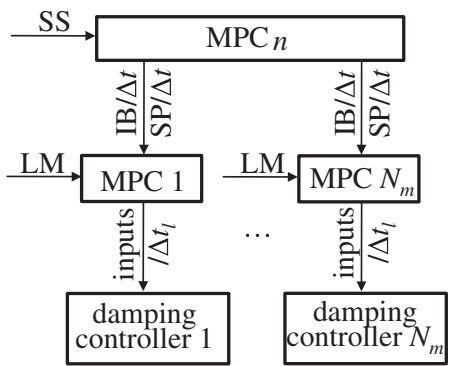

LM: local measurements; SP: set-points; SS: subsystem states; IB: input bases;

Fig. 2. Hierarchical MPC scheme of subsystem $n$. $p \hat{\delta}=K_{1} \Delta \omega$

$p \omega=K_{2} T_{m}-K_{3} T_{e}-K_{4} \Delta \omega$

$p E_{q}^{\prime}=K_{5} E_{f}-K_{6} E_{q}^{\prime}-K_{7} i_{d}+K_{8} \psi_{1 d}$

$\psi_{d}^{\prime \prime}=K_{9} E_{q}^{\prime}+K_{10} \psi_{1 d}$

$p E_{d}^{\prime}=-K_{11} E_{d}^{\prime}-K_{12} i_{q}+K_{13} \psi_{1 q}$

$\psi_{q}^{\prime \prime}=K_{14} E_{d}^{\prime}+K_{15} \psi_{1 q}$

$p E_{f}=-K_{16} E_{f}+K_{17} V_{r}$

$p V_{r}=-K_{18} V_{r}-K_{19} p R_{f}+K_{20}\left(V_{r e f}-V_{t}-V_{p s s}\right)$

$p R_{f}=-K_{21} R_{f}+K_{22} E_{f}$

$V_{\text {pss }}=G(p s s) \omega ; P_{\text {mech }}=G(\operatorname{tg}) \omega$,

where $p$ is the differential operator, $E_{q}^{\prime}$ and $E_{d}^{\prime}$ are $q$ axis and $d$ axis component of stator voltage behind sub-transient reactance, $\psi_{q}^{\prime \prime}$ and $\psi_{d}^{\prime \prime}$ are $q$ axis and $d$ axis air gap flux linkage, $\psi_{1 q}$ and $\psi_{1 d}$ are amortisseur circuit flux linkages of $q$ axis and $d$ axis, $E_{f}$ is field voltage, $V_{r}$ and $R_{f}$ are exciter states, $V_{\text {ref }}$ is voltage reference of exciter, $V_{t}$ is terminal voltage of generator, $V_{p s s}$ is PSS output, $P_{\text {mech }}$ is mechanical power, $G(p s s)$ and $G(t g)$ are transfer functions of PSS and turbine governor, $K_{1}, K_{2} \ldots K_{22}$ are coefficients. Detailed models are given in [28]. Using these models, a lower MPC controller calculates a supplementary signal for its PSS to reach the objective of making the corresponding generator run at base frequency, which is defined as (subscript $m$ refers to generator $m$ ):

$\min _{u_{m}[\cdot]}\left\{\sum_{i=0}^{N_{i}-1}\left(\hat{y}_{m}[k+i+1 \mid k]-y_{r . m}\right)^{2}\right\}$

subject to:

$u_{\text {min.m }} \leqslant u_{m}[k+i \mid k] \leqslant u_{\text {max.m. }}, \quad i=0,1,2, \ldots, N_{i}-1$.

$z_{\text {min.m }} \leqslant z_{m}[k+i+1 \mid k] \leqslant z_{\text {max.m }}, \quad i=0,1,2, \ldots, N_{i}-1$.

An MPC controller installed on a TCSC calculates, at every refreshing time, the active power increment of its tie-line and assumes that there will be a negative increment with same size at the next refreshing time. So, it can calculate line reactances from:

$P=\frac{V_{1}^{2} R-V_{1} V_{2} R \cos \theta+V_{1} V_{2} X \sin \theta}{R^{2}+X^{2}}$

at two refreshing times, and then gets the corresponding MPC inputs for the TCSC. In Eq. (14) $P$ is active power; $V_{1}$ and $V_{2}$ are voltage magnitudes at both ends; $\theta$ is angle difference of voltages at both ends; $R+j X$ is line impedance.

\subsubsection{Coupling between the two layers of MPC}

Two ways of coupling are investigated (see Fig. 2).

- Input base coupling: every $\Delta t$ seconds, the area-wise MPC controller collects subsystem states and calculates optimal inputs for controllers under its authority. It sends the inputs to device-level MPC controllers as their decision bases. Every $\Delta t_{l}$ seconds, each device-level MPC controller computes an input correction according to its local measurements and control objective, and combines the correction with input base as the supplementary control input of its damping device.

- Set-points coupling: the upper MPC controller solves an optimization problem and sends predicted system dynamics, angular speeds and line powers over a future time horizon, to lower MPC controllers as their set-points. The lower MPC controllers calculate the optimal supplementary inputs for damping controllers in order to drive the controlled outputs to reach set-points given by the upper MPC controller. 


\subsubsection{Coordination between lower MPC controllers}

The controlled output of a lower MPC controller depends not only on its states and input, but also on states and controls in the rest of a system. For a lower MPC controller, system variables and controls can be divided into two categories: internal and external. During a refreshing interval of upper MPC, it considers external variables and controls as constant, and uses them as simulation scenarios reflecting the anticipated influence of external variables and controls. The model of a lower-level MPC controller $m$ of subsystem $n$ is thus represented as follows:

$$
\begin{aligned}
x_{m}[k+1 \mid k]= & A_{m} x[k \mid k]+B_{m} u[k \mid k]=\left[A_{m, m}, A_{m, \text { ext }}\right]\left[\begin{array}{c}
x_{m}[k \mid k] \\
x_{\mathrm{ext}_{m}}[k \mid k]
\end{array}\right] \\
& +\left[B_{m, m}, B_{m, \mathrm{ext}}\right]\left[\begin{array}{c}
u_{m}[k \mid k] \\
u_{\mathrm{ext}_{m}}[k \mid k]
\end{array}\right]=A_{m, m} x_{m}[k \mid k]+B_{m, m} u_{m}[k \mid k] \\
& +A_{m, \mathrm{ext}} x_{\mathrm{ext}_{m}}[k \mid k]+B_{m, \mathrm{ext}} u_{\mathrm{ext}_{m}}[k \mid k]
\end{aligned}
$$

where $x_{m}$ is the vector of internal state variables; $u_{m}$ is the vector of internal controls; $A_{m}$ and $B_{m}$ are the parts of $A$ and $B$ that are relative to $x_{m} ; x_{\text {ext }_{m}}$ is the vector of state variables external to controller $m$, and $u_{\text {ext }}$ is the external control vector. The external variables and controls for the local MPC are considered constant during one refreshment period of the upper MPC, and they are hence fixed at the values computed at the previous step by the upper MPC.

\section{Test system and simulation conditions}

The system of Fig. 3 is used to test the proposed MPC schemes. Fig. 4 illustrates two types of local controllers considered in this work and the way supplementary controls computed by the proposed schemes are brought to local controllers. Power System Toolbox (PST) $[1,28]$ is used to simulate system response and to derive the linearized state space model. A TCSC is installed between buses 69 and 70, and there is a PSS on each generator. The system is composed of two areas: A1 and A2, which are connected to each other by the tie-lines 1-2 and 8-9, the latter being equipped with a TCSC. In tests presented in this work, a temporary three-phase short-circuit to ground at bus 1 (cleared by opening the tie-line 1-2 followed by its reconnection after a short delay) causes oscil- lations between areas A1 and A2. As shown in Fig. 5 (dashed lines), the temporal evolution over a period of 20s of the power flow through line 1-2 and the angular speed of generator 1 corresponds to poorly damped oscillations.

The MPC state vector $x$ includes generator, exciter, PSS, turbine governor and TCSC states. Output variables are angular speeds. The input $u$ is a vector of supplementary inputs for PSSs and TCSC, which is constrained to $-0.1 \leqslant u \leqslant 0.1$. A time step $\delta=0.005 \mathrm{~s}$ is used to formulate the MPC dynamics described by Eq. (3). Prediction horizon is set to $H_{p}=15$ discrete steps of $\Delta t=0.1 \mathrm{~s}$ (i.e. a prediction horizon of $1.5 \mathrm{~s}$ ) and a control horizon of $H_{u}=3$ steps (i.e. $u$ is optimized over the first $0.3 \mathrm{~s}$ and kept constant beyond). In the objective function Eqs. (5) and (9), all deviations of the predicted outputs from references are weighted uniformly and independently, i.e. $Q$ and $Q_{n}$ are the identity matrices. To assess the power system response with and without MPC controllers, it is simulated over a period of $20 \mathrm{~s}$.

\section{Analysis of the dynamic performances}

Subsequently, only the effects of different control schemes and assumptions on the tie-line 1-2 power flow and on the angular speed of generator 1 are shown. These are indeed representative of the overall dynamics of the system.

\subsection{Performance of the centralized MPC scheme}

\subsubsection{Baseline results in ideal conditions}

Ideal conditions are meant complete state observability and controllability, with SE error and delays neglected. Controlled system response is given in Fig. 5, in solid lines. Compared with the system response without MPC, settling time is decreased to approximately $10 \mathrm{~s}$. Fig. 6 shows the control signals computed by the MPC scheme for a representative PSS and the TCSC (first $5 \mathrm{~s}$ ).

\subsubsection{Results with SE errors and delays}

SE errors are simulated as an additive random noise. Uniformly distributed pseudorandom errors in the range from $-10 \%$ to $+10 \%$ are superimposed on the exact states. The idea is to test perfor-

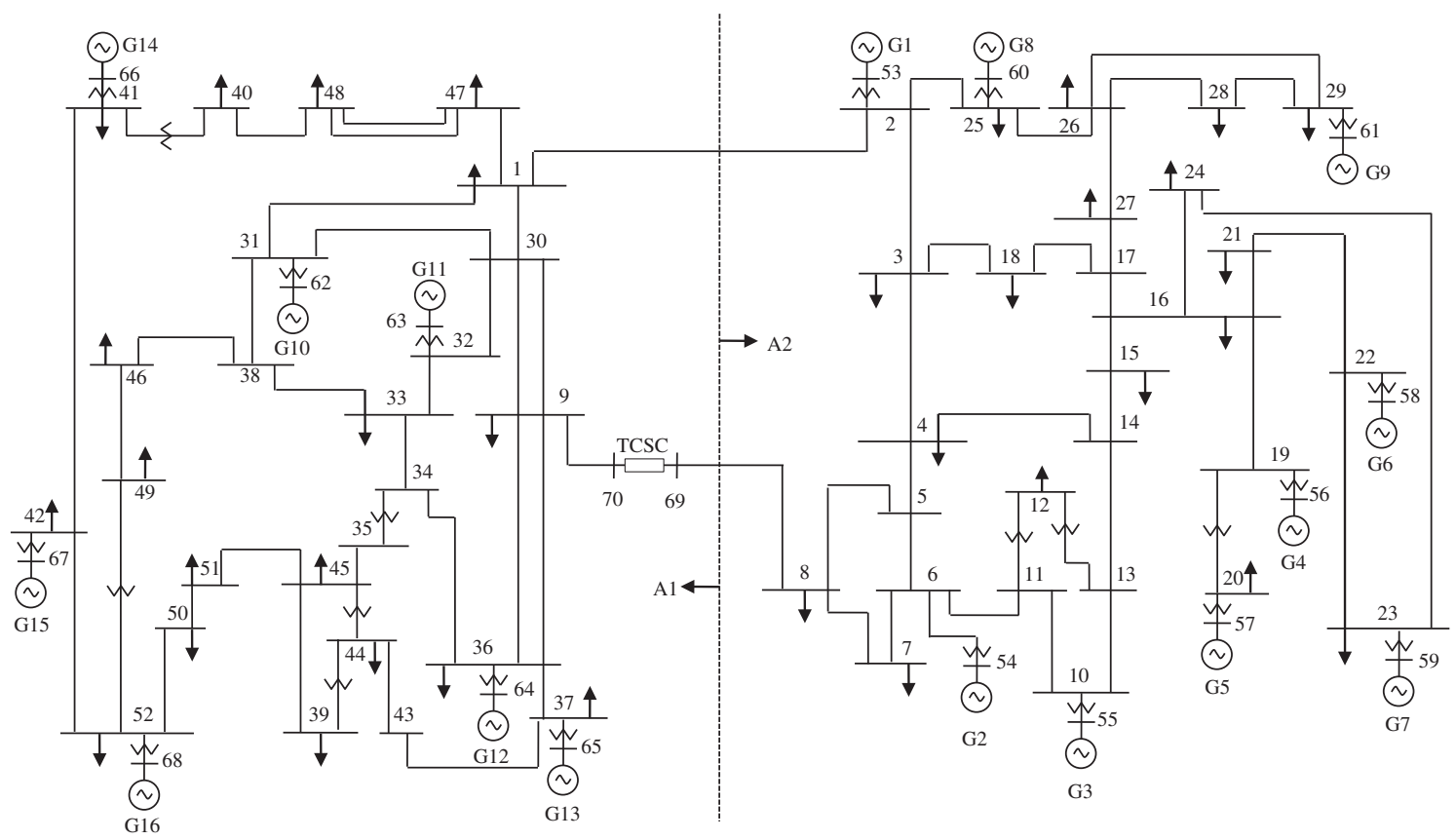

Fig. 3. 16 generators 2 areas 70 buses test system. 


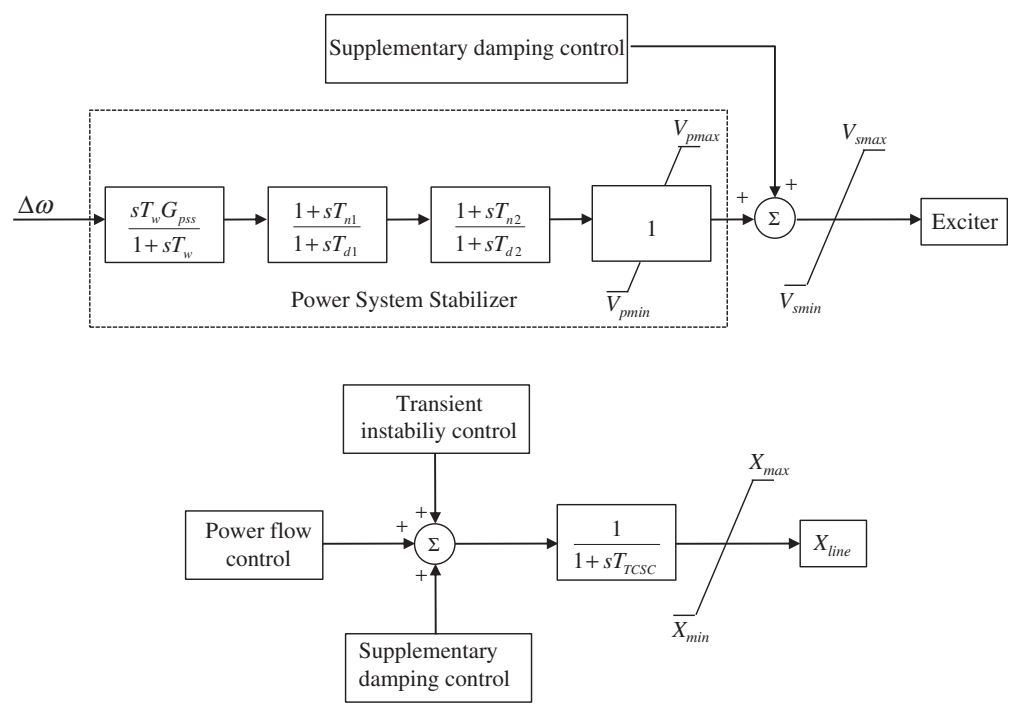

Fig. 4. Supplementary control inputs: PSS (top), TCSC (bottom).

mances of the control in presence of relatively high errors (maximum error in a state variable is $\pm 3 * \sigma$ with $\sigma=3.3 \%$, and these measurement standard deviations of measurements are revealed to be realistic even in case of PMUs since their accuracy is impacted by existing measurement equipments such as measurement
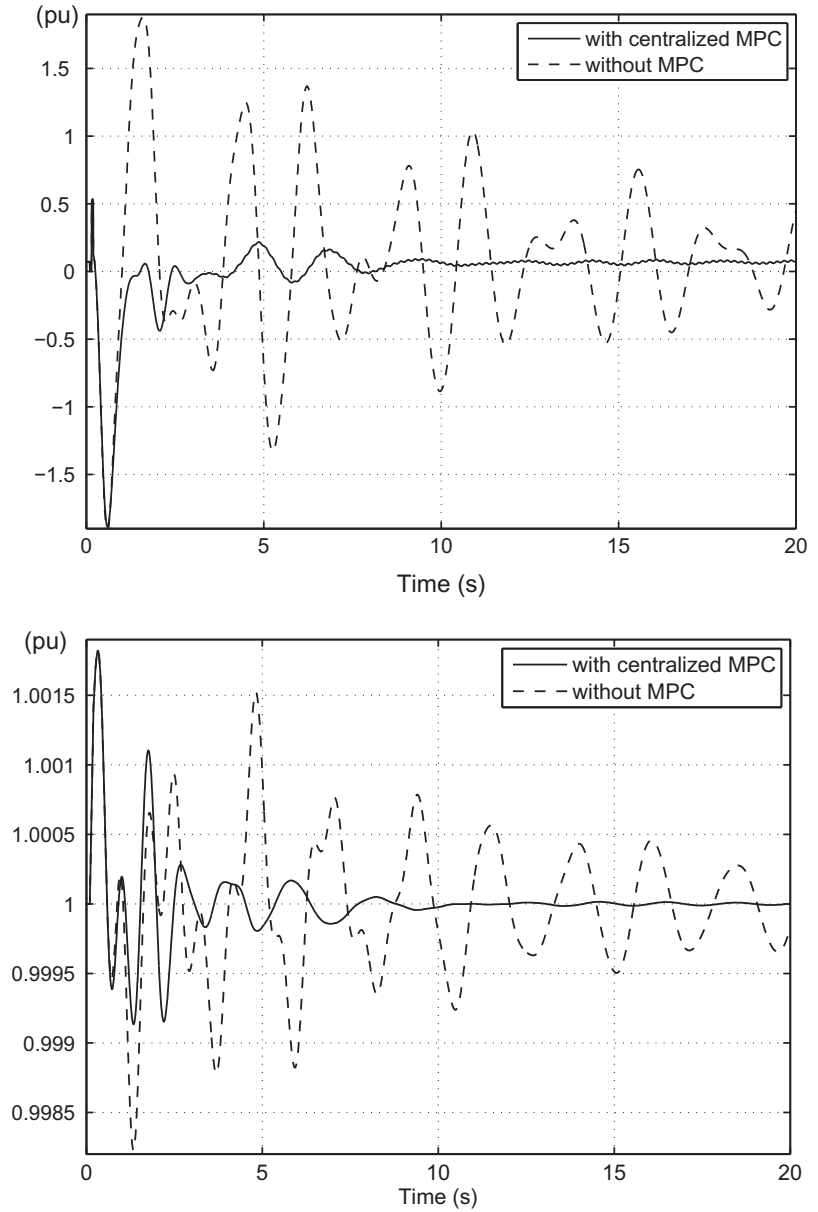

Fig. 5. Active power of line 1-2 (top), angular speed of generator 1 (bottom) transformers [30]). The results are shown in Fig. 7. As expected, inaccuracies introduced by SE errors affect the performances of the MPC scheme in terms of magnitude of oscillations and settling time. On the other hand, it is observed that the correction of SE errors considerably improves the control performances.
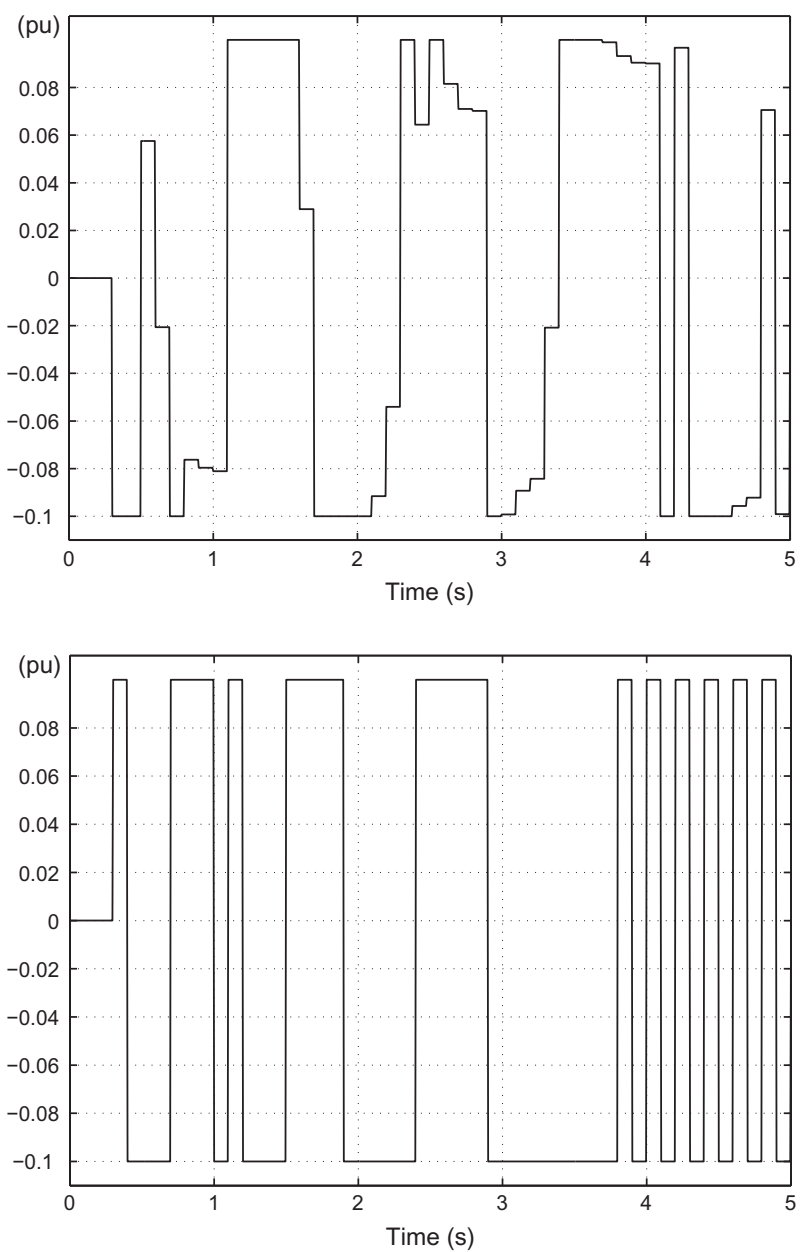

Fig. 6. Centralized MPC signals: PSS of generator 1 (top), TCSC (bottom) 
Time delays in computing and sending controls signals to LCs may vary in practice. There are two possibilities [6]: to apply controls as soon as they are available or after a specific time interval. As explained before, the latter option is considered. A common delay of $\tau=0.05 \mathrm{~s}$ in measuring, computing and sending control signals to all available LCs is assumed. The choice of this delay is based on observations presented in [31].

System response under this assumption is shown in Fig. 8 (SE errors are modeled in the simulations and corrected in the used MPC schemes). It is clear that the performances taking into account delays are worse than those with only SE errors, but still quite superior to those without MPC.

\subsection{Performance of the distributed MPC scheme}

In the distributed scheme, one area-wise MPC controller is used in each one of the two areas A1 and A2, while the TCSC is assigned to area A1 as one of its resources. MPC1 calculates supplementary MPC signals for PSS10-16 and TCSC; MPC2 is responsible for PSS19. Results with distributed MPC in ideal conditions have already been presented in [24]. Here, the results considering $\pm 10 \%$ SE errors and a $0.05 \mathrm{~s}$ delay are presented.

Controlled and uncontrolled system responses are shown in Fig. 9. Compared with the system response without MPC it is clear that this (trivial) distributed MPC scheme effectively damps the oscillations. In the condition only considering SE errors, the centralized MPC is however superior to the distributed one. But when $0.05 \mathrm{~s}$ delay is introduced, it is observed that the distributed scheme yields a slightly better control effect. This is due to chosen decomposition method. The system is decoupled by substituting exchange powers for two equivalent loads which absolute values are equal to exchange powers at steady state. So, linearized models calculated in this condition contain the information about these powers. In addition to the explicit control objective of Eq. (9), the area-wise MPCs thus have the implicit objective to restore exchange powers to the steady state values, and this actually helps to drive the system to steady state.

\subsection{Performance of the hierarchical MPC scheme}

MPC controllers are installed on each PSS and the TCSC. The upper MPC controllers use the same simulation parameters as distributed MPC. The lower MPC controllers refresh decisions at discrete steps of $\Delta t_{l}=0.01 \mathrm{~s}$ and use a control horizon $H_{u l}=5 \mathrm{such}$

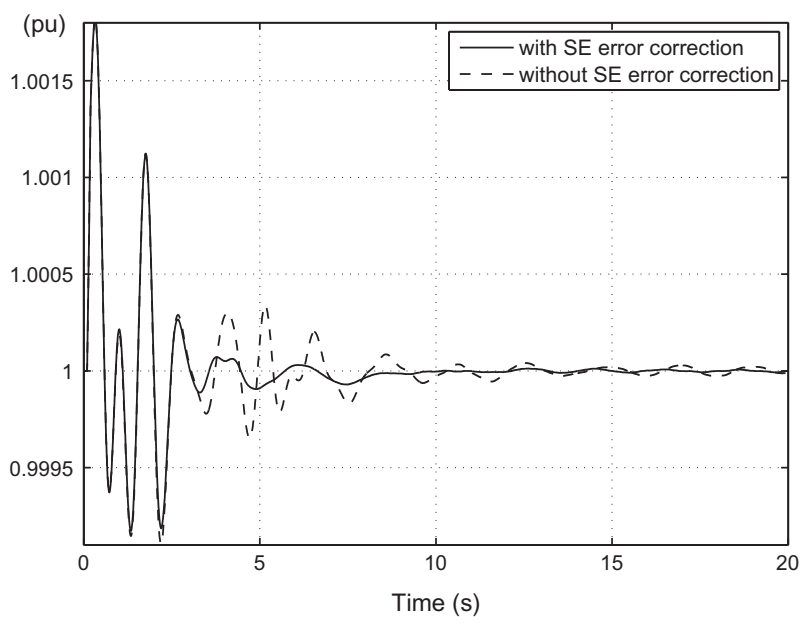

Fig. 7. Angular speed of generator 1 with centralized MPC involving SE errors.

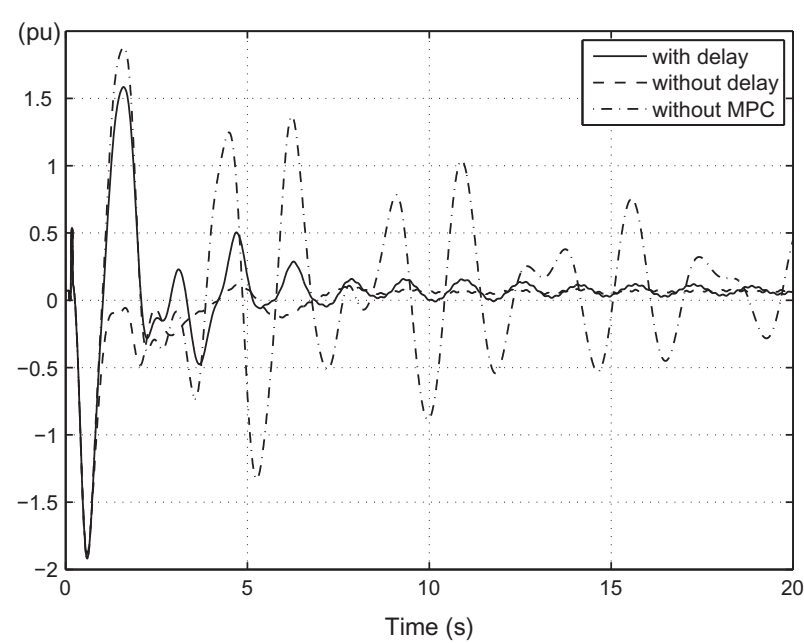

Fig. 8. $P_{1-2}$ with centralized MPC involving SE errors and $0.05 \mathrm{~s}$ delay.
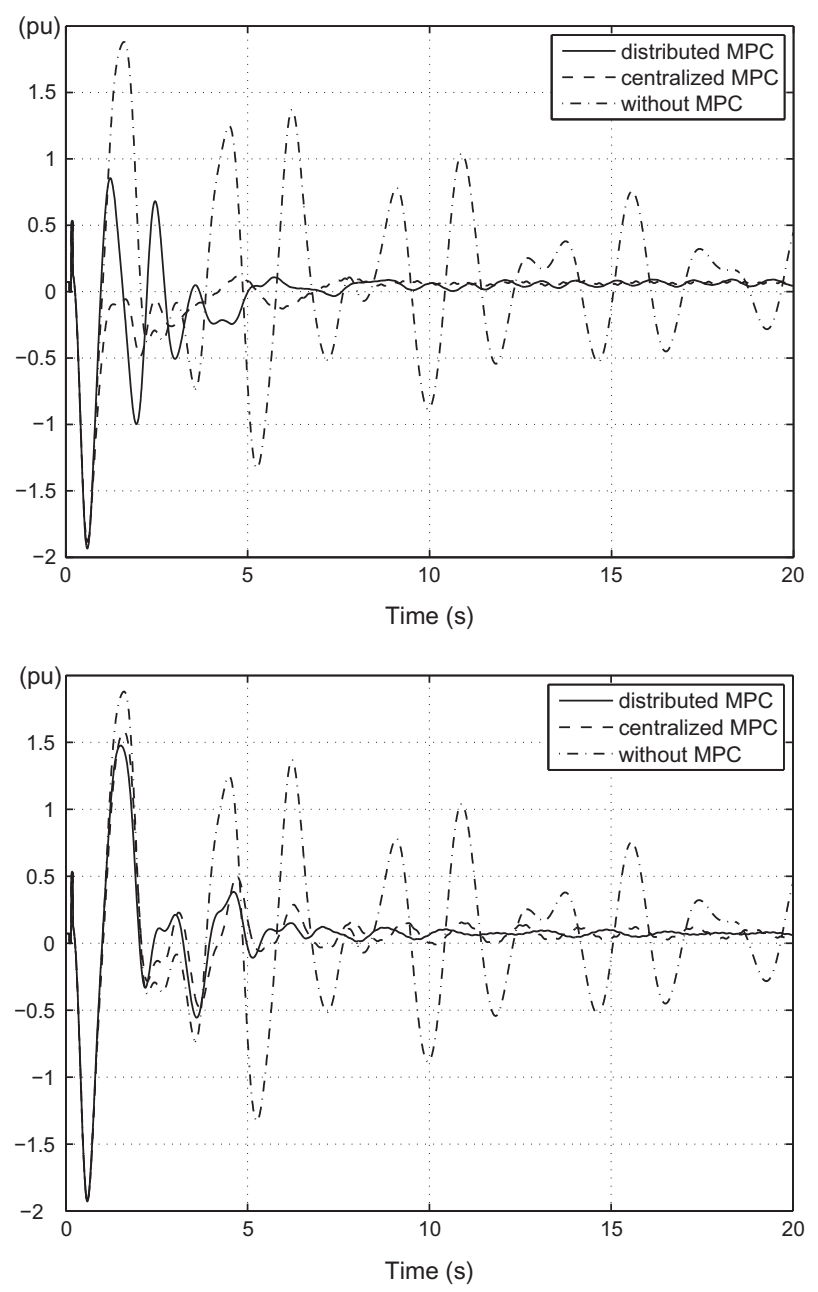

Fig. 9. $P_{1-2}$ with distributed MPC: with SE errors (top), with SE errors and $0.05 \mathrm{~s}$ delay (bottom).

steps. The prediction horizons $H_{p l}$ are set to 60 (A1) and 40 (A2). Fig. 10 shows controlled system response with the same SE errors and delays as before. Compared with the solely distributed MPC scheme, the hierarchical one further slightly improves control effects. 
Next, the hierarchical MPC is compared to the distributed scheme in terms of robustness, with respect to the following five aspects:

1. Increasing the refreshing step $\Delta t$ of area-wise controllers to $0.2 \mathrm{~s}$.

2. Simulating the complete failure of the MPC of A1 during the first $5 \mathrm{~s}$.

3. Incomplete measurements by assuming that only states of generators $10-13$ in area $A 1$ and generators $1-5$ in area $A 2$ are available for upper MPC controllers.

4. Communication failure between upper MPC controllers and local damping devices or MPC controllers. It is assumed that PSSs or lower MPC controllers on generators 1-4 and 10-13 cannot receive the input signals from the upper MPC controllers.

5. Different operation conditions through comparison of distributed MPC and hierarchical MPC with topology change (tripping tie-line 1-2) and flow change (change in flows over two tielines from $0.07 \mathrm{pu}$ to $0.4 \mathrm{pu}$ in the line $1-2$ and from $0.38 \mathrm{pu}$ to $0.14 \mathrm{pu}$ in the line $8-9$ ).

The results are shown in Figs. 11-14. These results show that hierarchical MPC offers excellent robustness since it accommo-

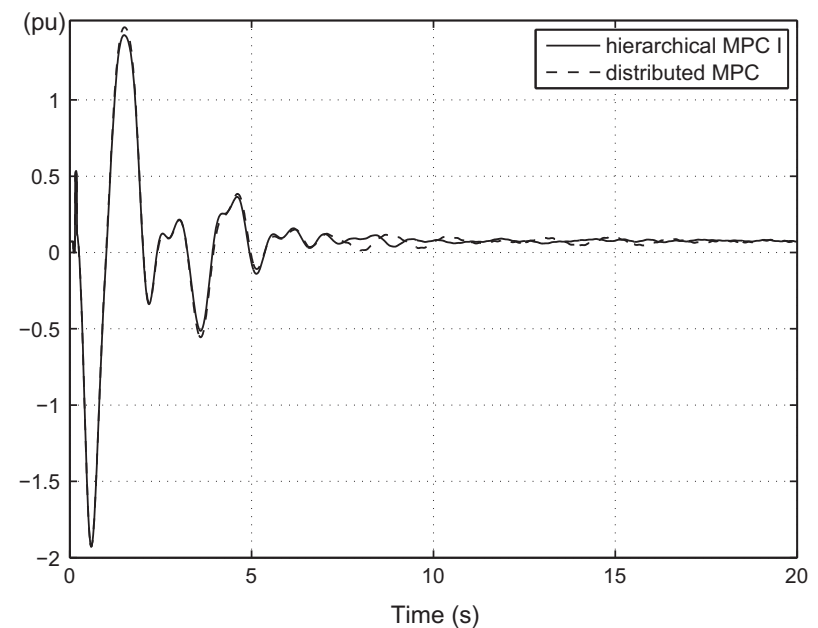

Fig. 10. $P_{1-2}$ with hierarchical and distributed MPC

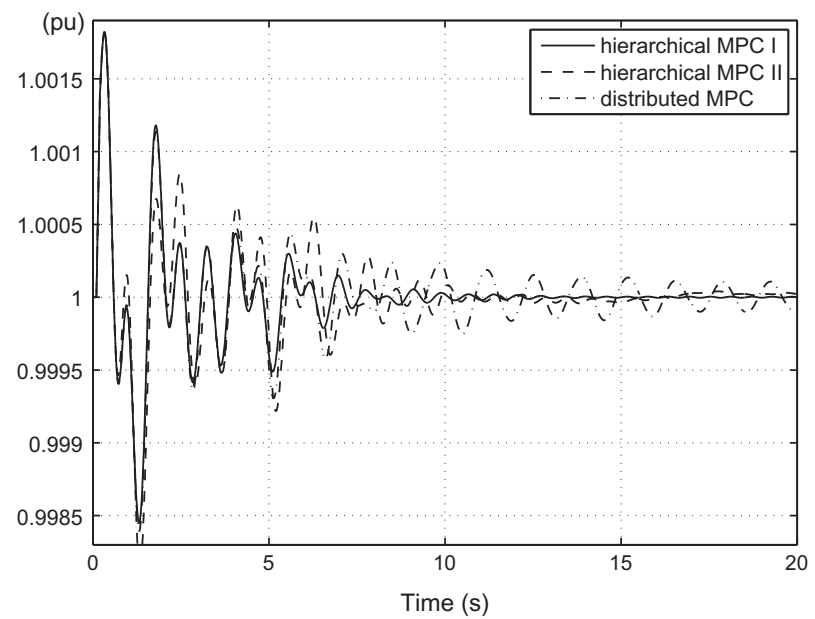

Fig. 11. Angular speed of generator 1 using refreshing interval of $0.2 \mathrm{~s}$. dates larger refreshing intervals, tolerates controller and communication failures, and works with incomplete measurement and in different operating conditions.

Fig. 11 compares two proposed couplings of the hierarchical MPC. Hierarchical MPC I means input base coupling while hierar-

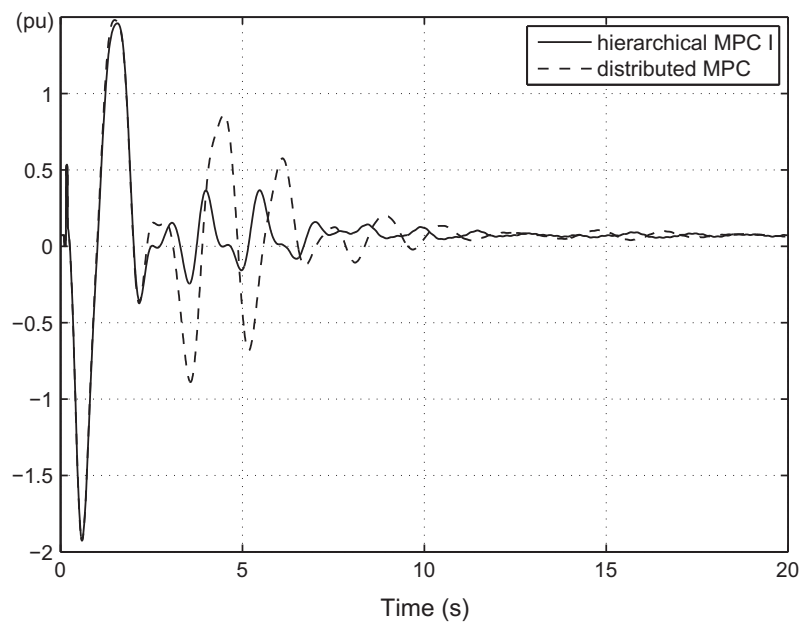

Fig. 12. $P_{1-2}$ with an upper MPC1 failure.
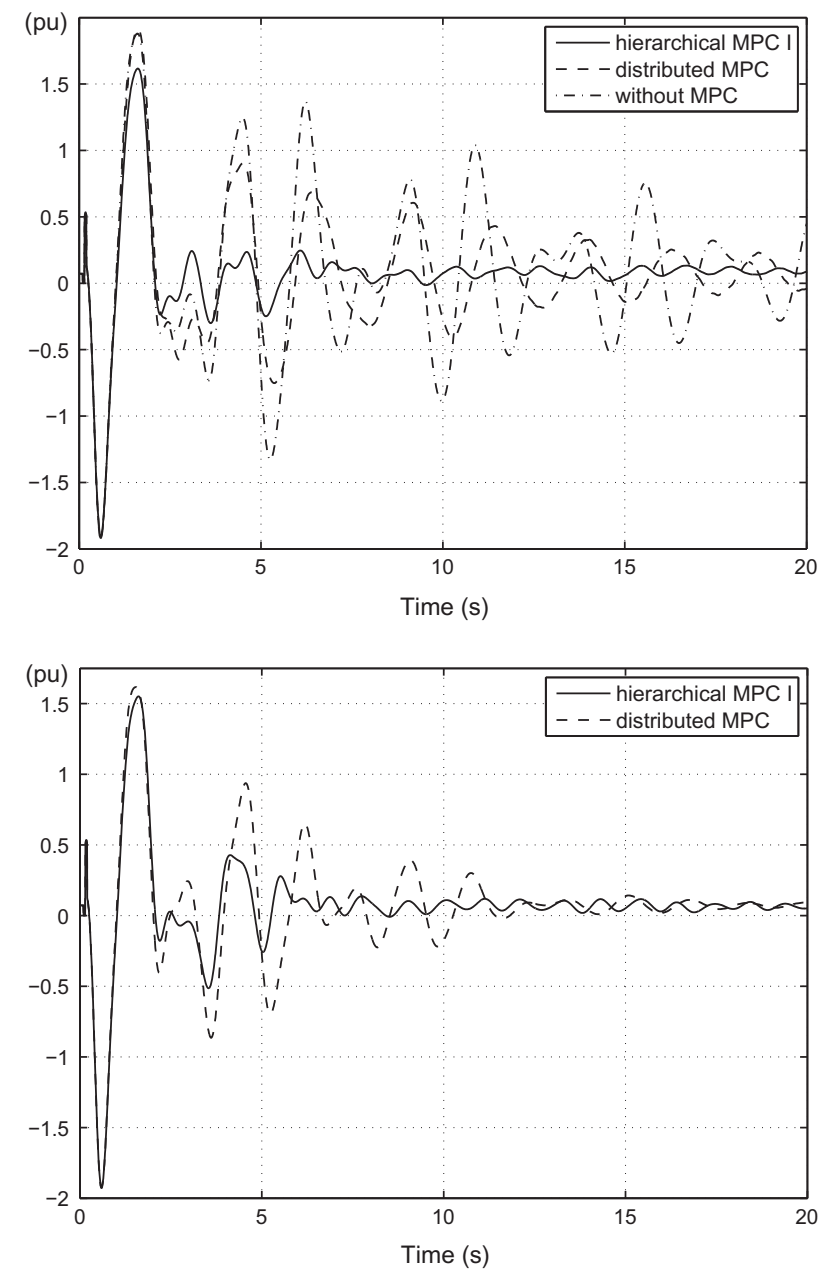

Fig. 13. $P_{1-2}$ with incomplete measurement (top), and communication failure (bottom). 


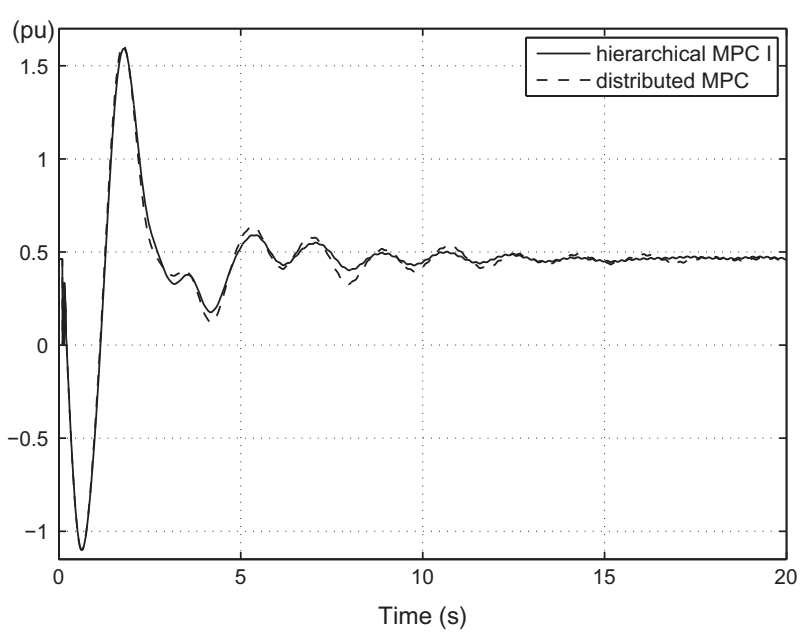

Fig. 14. $P_{1-2}$ with topology and flow changes.

chical MPC II means set-points coupling. In the hierarchical MPC II, large refreshing interval makes lower MPC controllers only use predicted values with larger errors as their set-points because prediction precision becomes worse in time. In the hierarchical MPC I, lower MPC controllers use rated angular speeds as their control objectives and continuously adjust their input corrections following system dynamics, thus yielding a slightly better performance.

\subsection{Computational considerations}

The computational efficiency of the hierarchical MPC is checked on a i7-3610 processor and 8G RAM. The MPC optimizations of Eqs. (5), (9) and (12) are solved by an Active Set method built in the Quadprog function of MATLAB. It takes from 10 to $20 \mathrm{~ms}$ for the upper MPC controller, and from 3 to $5 \mathrm{~ms}$ for the lower MPC controllers, to complete one optimization.

\section{Conclusion}

This paper investigated the feasibility of applying MPC to damp wide-area electromechanical oscillations in large-scale power systems. A centralized MPC scheme based on linearized discrete-time state space model of a system, is used as a baseline. A distributed MPC scheme is introduced in which each MPC controller solves its optimization subproblem using a detailed model of its own area and a rough model of the remaining areas. It then sends the inputs to local damping controllers under its responsibility. The common control target is used to implicitly coordinate these MPC controllers. Finally, a two-level hierarchical multi-area MPC scheme is introduced with the aim to enhance control robustness. The upper MPC controllers optimize inputs of damping controllers in their own area and send them to the lower MPC controllers as their input bases. Using local models and measurements, the lower MPC controllers correct these input bases.

The performances of the proposed control schemes are tested using a 70-bus test system, both in ideal conditions and considering state estimation errors and communication delays. Simulation results show that the distributed scheme appears to be a viable control strategy for large-scale systems while the hierarchical MPC further improves control effects of the distributed one, and offers at the same time much better robustness.

This paper focused on exploring feasibility of MPC for improved damping of electromechanical oscillations. Of course, several practical issues deserve further investigation and further work will be primarily focused on:
- Testing hierarchical MPC using models of larger real-life power systems.

- Consideration of some other combinations of SE errors and delays (different for different measurements and controls).

- Studying the effects and testing the performances of the proposed control schemes in the presence of ambient oscillations such as continuous load or dispersed generation variations.

While MPC, being a closed-loop control scheme, has some intrinsic level of robustness to modeling errors, it nevertheless relies on the use of a correct dynamic model of the system. Within the context of power system oscillation damping, load-dynamics, and dynamics of renewable and dispersed generation may have a significant impact on the system behavior; since the composition of the load and dispersed generation may change significantly from one period of time to another (e.g. intra-daily, and seasonal effects driven by weather conditions) the system dynamics at a particular moment may not be well enough approximated by the model computed from the available data in TSO control centers to yield satisfactory performances of any one of the proposed MPC schemes. Another aspect of future work therefore should be devoted to real-time model identification and to methods able to carry out model-free learning of supplementary controls.

\section{Acknowledgments}

This paper presents research results of the Belgian Network DYSCO, funded by the Interuniversity Attraction Poles Programme, initiated by the Belgian State and of the PASCAL Network of Excellence funded by the European Commission. The scientific responsibility rests with the authors.

\section{References}

[1] Rogers G. Power system oscillations. Norwell (Massachusetts, USA): Kluwer Academic Publishers; 2000.

[2] Grebe E, Kabouris J, Barba SL, Sattinger W, Winter W. Low frequency oscillations in the interconnected system of continental Europe. In: Proc of IEEE Power and Energy Society General Meeting 2010. Minneapolis, MN; July, 2010.

[3] Grund CE, Paserba JJ, Hauer JF, Nilsson S. Comparison of Prony and eigenanalysis for power system control design. IEEE Trans Power Syst 1993;8(3):964-71.

[4] Sakis Meliopoulos AP, Cokkinides GJ, Galvan F, Myrda P. Delivering accurate and timely data to all: model-based substation automation applications for advanced data availability. IEEE Power Energy Mag 2007:5(3):74-86.

[5] Kamwa I, Grondin R, Hebert Y. Wide-area measurement based stabilizing control of large power systems - a decentralized/hierarchical approach. IEEE Trans Power Syst 2001;16(1):136-53.

[6] Maciejowski JM. Predictive control with constraints Harlow. England: Prentice Hall; 2002.

[7] Qin SJ, Badgwell TA. A survey of industrial model predictive control technology. Control Eng Practice 2003;11:733-64.

[8] Camponogara E, Jia D, Krogh BH, Talukdar S. Distributed model predictive control. IEEE Control Syst Mag 2002;9(1):44-52.

[9] Scattolini R. Architectures for distributed and hierarchical model predictive control: a review. J Process Control 2009;19:723-31.

[10] Monticelli A, Pereira MVF, Granville S. Security-constrained optimal power flow with post-contingency corrective rescheduling. IEEE Trans Power Syst 1987;2(1):175-80.

[11] Marinescu B, Bourles H. Robust predictive control for the flexible coordinated secondary voltage control of large-scale power systems. IEEE Trans Power Syst 1999:14(4):1262-8.

[12] Talukdar S, Jia D, Hines P, Krogh BH. Distributed model predictive control for the mitigation of cascading failures. In: Proc of 44th IEEE conference on decision and control. Seville, Spain; 2005. p. 4440-5.

[13] Otomega B, Marinakis A, Glavic M, Van Cutsem T. Model predictive control to alleviate thermal overloads. IEEE Trans Power Syst 2007;22(8):1384-5.

[14] Larsson M, Karlsson D. Coordinated system protection scheme against voltage collapse using heuristic search and predictive control. IEEE Trans Power Syst 2003;18(3):1115-22.

[15] C Jin L, Kumar R, Elia N. Model predictive control-based real-time power system protection schemes. IEEE Trans Power Syst 2010;25(2):988-98

[16] Glavic M, Hajian M, Rosehart W, Van Cutsem T. Receding-morizon multi-step optimization to correct nonviable or unstable transmission voltages. IEEE Trans Power Syst 2011;26(3):1641-50. 
[17] Ford JJ, Ledwich G, Dong ZY. Efficient and robust model predictive control for first swing transient stability of power systems using flexible AC transmission systems devices. IET Generat Trans Distribut 2008;2(5):731-42.

[18] Chaudhuri B, Majumder R, Pal BC. Application of multiple model adaptive control strategy for robust damping of interarea oscillations in power system. IEEE Trans Control Syst Technol 2004;12(5):727-36.

[19] Majumder R, Chaudhuri B, Pal BC. A probabilistic approach to model-based adaptive control damping of interarea oscillations in power system. IEEE Trans Power Syst 2005;20(1):367-74.

[20] Short TA, Pierre DA, Smith JR. Self-tuning generalized predictive control for switched capacitor damping of power system oscillations. In: Proc of 28th IEEE conference on decision and control, vol. 2. Tampa, FL; 1989. p. 1847-52.

[21] Johansson NP, Nee HP, Angquist L. An adaptive model predictive approach to power oscillation damping utilizing variable series reactance FACTS devices. In: Proc of 41st int universities power engineering conference (UPEC), vol. 2. Newcastle, UK; 2006. p. 790-4.

[22] Wang L, Cheung H, Hamlyn A, Cheung R. Model predictive adaptive control of inter-area oscillations in multi-generator power systems. In: Proc of the IEEE PES general meeting. Calgary, Canada; 2009. p. 1-7.

[23] Wang D, Glavic M, Wehenkel L. A new MPC scheme for damping wide-area electromechanical oscillations in power systems. In: Proc of the 2011 IEEE PES powertech. Trondheim, Norway; 2011.

[24] Wang D, Glavic M, Wehenkel L. Distributed MPC of wide-area electromechanical oscillations of large-scale power systems. In: Proc of the 16th intelligent system applications to power systems (ISAP). Crete, Greece; 2011.

[25] Venkat AN, Hiskens IA, Rawlings JB, Wright SJ. Distributed MPC strategies with application to power system automatic generation control. IEEE Trans Control Syst Technol 2008;16(6):1192-206.

[26] Roshany-Yamchi S, Cychowsky M, Negenborn RR, De Schutter B, Delaney K, Connell J. Kalman filter-based distributed model predictive control of largescale multi-rate systems: application to power networks. IEEE Trans Control Syst Technol 2013;23(1):27-39.

[27] Okou F, Dessaint LA, Akhrif O. Power systems stability enhancement using a wide-area signals based hierarchical controller. IEEE Trans Power Syst 2005;20(3):1465-77.

[28] Chow JH, Cheung KW. A toolbox for power system dynamics and control engineering education and research. IEEE Trans Power Syst 1992;7(4):1559-64.

[29] Motee N, Rodsari BS. Optimal partitioning in distributed model predictive control. In: Proc of the 2003 American control conference colorado. USA; June, 2003. p. 5300-5.

[30] Meliopoulos APS, Cokkinides GJ, Galvan F, Fardanesh B. Distributed state estimator-advances and demonstration. In: Proc 2008 41st Hawaii int conf system sciences. Waikoloa, USA; 2008.

[31] Wu H, Tsakalis KS, Heydt GT. Evaluation of time delay effects to wide-area power system stabilizer design. IEEE Trans Power Syst 2004;20:1935-41. 